\title{
BMJ Open Supported self-management in community stroke rehabilitation: what is it and how does it work? A protocol for a realist evaluation study
}

\author{
Lisa Kidd (D) , ${ }^{1}$ Julie Duncan Millar, ${ }^{1}$ Helen Mason, ${ }^{2}$ Terry Quinn, ${ }^{3}$ \\ Katie I Gallacher (1) , ${ }^{4}$ Fiona Jones, ${ }^{5}$ Rebecca J Fisher, ${ }^{6}$ Therese Lebedis, ${ }^{7,8}$ \\ Mark Barber, ${ }^{8,9}$ Katrina Brennan, ${ }^{8,10}$ Mark Smith ${ }^{8,11}$
}

To cite: Kidd L, Millar JD, Mason $\mathrm{H}$, et al. Supported self-management in community stroke rehabilitation: what is it and how does it work? A protocol for a realist evaluation study. BMJ Open 2022;12:e55491. doi:10.1136/ bmjopen-2021-055491

- Prepublication history for this paper is available online. To view these files, please visit the journal online (http://dx.doi. org/10.1136/bmjopen-2021 055491).

Received 13 July 2021 Accepted 12 November 2021

Check for updates

(C) Author(s) (or their employer(s)) 2022. Re-use permitted under CC BY-NC. No commercial re-use. See rights and permissions. Published by BMJ.

For numbered affiliations see end of article.

Correspondence to

Dr Lisa Kidd;

lisa.kidd@glasgow.ac.uk

\section{ABSTRACT}

Introduction A growing evidence base demonstrates the effectiveness of supported self-management in stroke for stroke survivors and their families. However, there is significant variation in its implementation in community stroke care and little understanding about how supported self-management works and is delivered across different settings, models used and contexts of community stroke rehabilitation.

Methods and analysis Using a mixed method, realist approach across two phases, this protocol describes a study on community-based supported self-management. The aim is to identify the mechanisms and outcomes of supported self-management in stroke and to understand how supported self-management is implemented in different contexts of community stroke rehabilitation. Phase 1 involves (1) a realist synthesis, (2) a scoping and mapping of current community rehabilitation settings and (3) a Q-methodology study to develop initial programme theories about how community-based supported selfmanagement works, for whom and in what contexts. Phase 2 involves realist informed interviews/focus groups with stroke survivors, community rehabilitation practitioners and team managers from across Scotland to test and refine programme theories and an explanatory model for how supported self-management works across different contexts of community-based stroke rehabilitation.

Ethics and dissemination Ethical approval and R\&D approvals have been granted from East of Scotland Research Ethics Committee (REC reference number: 19/ ES/0055) and participating NHS boards. An understanding of how, for whom and in what contexts community-based supported self-management works will help to strengthen its delivery in practice. Such an understanding will enable the design of context-specific recommendations for policy and practice that genuinely reflect the challenges in implementing supported self-management in community stroke care. Results will be disseminated to clinical partners working in community stroke rehabilitation, stroke survivors and families and to policymakers and third sector partners involved in the provision of long-term support for people affected by stroke.

PROSPERO registration number CRD42020166208.
Strengths and limitations of this study

- This is one of the first studies using realist evaluation to understand the mechanisms and contexts in which supported self-management in stroke works.

- The use of Q-methodology within a realist evaluation study is novel and valuable for helping to develop initial programme theories around how supported self-management works.

- Further research may be required to understand how the study findings and recommendations apply to the implementation of supported self-management in different contexts of stroke care (eg, acute care) and in different countries.

\section{INTRODUCTION}

Stroke is recognised as an acute event with complex and enduring physical, cognitive and psychosocial consequences that require effective, long-term support delivered in primary and community care. ${ }^{1-3}$ Supported self-management features as a core component of personalised care and rehabilitation in international long-term condition (LTC) management policies and frameworks, ${ }^{4-7}$ including for stroke survivors and their families. ${ }^{8}$ Supported self-management, particularly in stroke, is a poorly defined concept. ${ }^{10}$ Contemporary notions position this as being multidisciplinary approaches and interventions that aim to help people develop the skills, knowledge and confidence to manage the medical and emotional aspects of LTCs, multiple morbidities and treatment burden, and to maintain valued life roles in the context of their condition(s). ${ }^{11} 12$ This conceptualisation represents a move away from traditional views of self-management as being merely about education or compliance with professionally driven treatment plans and goals, to 
supporting people to live their lives in a personally meaningful way.

There is a growing evidence base highlighting the effectiveness of supported self-management in people with stroke. Systematic reviews, collectively summarising the findings of 40 primary studies on stroke self-management interventions, have reported statistically significant improvements in: quality of life, self-efficacy and a range of health-related behaviours including reduced use of health services, smoking and alcohol intake; recovery from disability; functional independence; and participation in activities of daily living. ${ }^{13-17} \mathrm{~A}$ recent randomised controlled trial of the 'Take Charge' programme, a community-based self-management intervention for stroke survivors in New Zealand, also reported improvements in physical health, activities of daily living and independence. ${ }^{18}$ However, significant heterogeneity exists across such interventions in stroke, which typically comprise components such as goal setting, decisionmaking, self-monitoring and support for living with life after stroke. ${ }^{16}$ Patient experience and qualitative data consistently report that supported self-management is valued by stroke survivors and their families, as well as stroke professionals. $^{19-23}$

While the positive impact of supported self-management in stroke is encouraging, the active ingredients for success or mechanisms of change in such interventions remains unclear. ${ }^{192}$ Several well-defined supported selfmanagement interventions in stroke exist, for example, Bridge $^{25}$ in the UK and Take Charge in New Zealand. ${ }^{18} 24$ However, our previous research and anecdotal evidence suggests that the diffusion and delivery of supported selfmanagement in practice requires organisational culture change within the healthcare system, rather than the simple implementation of a discrete and well-defined intervention with specific evaluation outcomes. ${ }^{20} 2526$ Supported self-management approaches have often been delivered according to a set of principles or ideas that fit with, or are in some way influenced by, the system or organisational context. As a result, there is variation in its delivery across stroke, particularly in community settings, ${ }^{27}$ and evidence that this provision does not always align with stroke survivor's self-management needs and priorities. ${ }^{28} 29$

It is plausible that supported self-management in stroke may work in different ways, or not at all, because of factors inherent in the geographical or organisational contexts or models of rehabilitation delivery in which it is being implemented. ${ }^{30}$ The implementation of supported self-management may also be influenced by different perspectives on, and interpretations of, supported self-management in stroke. ${ }^{26}$ Thus, although evidence suggests supported self-management in stroke is effective, to optimise and meaningfully tailor its delivery to stroke survivors' needs in the community, we need to learn more about how supported self-management works and how it is delivered in different settings of community rehabilitation. The proposed realist evaluation study aims to address this gap.

\section{STUDY AIMS AND OBJECTIVES}

\section{Aim}

To (1) identify the mechanisms and outcomes of community-based supported self-management and, (2) understand how supported self-management is implemented and 'what makes it work' in different contexts of community stroke rehabilitation from the perspectives of stroke survivors, community rehabilitation practitioners and team managers.

\section{Objectives}

1. To explore stroke survivors', community rehabilitation practitioners' and team managers' perceptions of the mechanisms and outcomes involved in supported selfmanagement in stroke.

2. To describe the structure, organisation and delivery of community-based supported self-management and the factors likely to influence how this is implemented in different contexts for people who have had a stroke.

3. To identify the context-mechanism-outcome relationships that explain how, for whom and in what contexts supported self-management works in community rehabilitation for people who have had a stroke.

\section{METHODS AND ANALYSIS \\ Study design}

The overarching framework for this study is Realist Evaluation, ${ }^{31}$ a form of theory-driven evaluation which acknowledges that interventions and programmes work differently in different circumstances or contexts. ${ }^{32}$ Realist evaluation aims to derive a deeper understanding of how, why and for whom interventions or programmes work $^{33}$; in this case, how community-based supported self-management in stroke works, for whom and why. Specifically, realist evaluation aims to uncover how the outcomes of an intervention are produced because a set of hidden mechanisms are triggered (or not) depending on how they interact with the characteristics of the context (eg, individual, interpersonal or organisational factors) in which the intervention is situated. ${ }^{33} 34$ Context, mechanism and outcome (CMO) configurations are a heuristic used by researchers to develop realist informed programme theories about how interventions are supposed to work which are then empirically tested and refined. ${ }^{35}$ Middle range theories, which help to explain patterns in social behaviour and social change at a more abstract or generalisable level ${ }^{33}$ are also applied to the refined programme theories in realist evaluation research to help provide an understanding of how types of intervention work in different types of circumstances. ${ }^{36}$ Table 1 provides a summary of definitions underpinning the research. 
Table 1 Summary of definitions underpinning the study

\section{Programme For the purposes of this project, we will refer to supported self-management as a programme}

Programme Programme theories are ideas or hypotheses about '...about how, and for whom, to what extent and in what theory contexts a programme might "work". ${ }^{32}$

$\mathrm{CMO}$

The Context-Mechanism-Outcome configuration is abbreviated to CMO. CMO is a heuristic or hypothesis important to building and refining theory in realist studies. The CMO configuration is used to demonstrate generative causality by unpacking how and why a programme works at a granular level. CMOs are embedded within programme theories.

Context Context tells us for whom and in what circumstances a programme may or may not work. Context can be thought of as the backdrop of a programme that enacts or inhibits the firing of a mechanism to produce an outcome. Context can include individual, interpersonal or organisational factors.

Mechanism Mechanisms tell us why a programme may or may not work. In realist evaluation, mechanisms are often hidden as they often include the reasoning or decisions that people make in response to resources offered by the programme. Thus, mechanisms are influenced by the context in which the programme is enacted. It is the combination of mechanism and context that generates outcomes.

\section{Outcome Outcomes can be intended or unexpected consequences of a programme.}

Middle range Middle range theories are a step more abstracted than programme theories and help to explore and explain theory how types of interventions work in different types of circumstances. ${ }^{36}$

In accordance with Pawson and Tilley's ${ }^{31}$ framework for realist evaluation research, the current study will be conducted across two phases:

- Phase 1: Developing and refining the initial programme theories of how supported selfmanagement in community rehabilitation works, for whom and in what contexts.

- Phase 2: Testing, synthesis and consolidation of the programme theories to develop a robust explanatory model of how community-based supported selfmanagement works, for whom and in what contexts.

Each phase involves several stages and, in accordance with a realist approach, is iterative and evolving in nature as the theories develop and require further exploration, testing and refinement. ${ }^{31} 37$ Figure 1 illustrates the planned phases of the study and depicts the iterative nature of the methodology through continuous cycles of literature reviewing that occur in developing and refining the programme theories. The study commenced in March 2019 and is due to be completed by December 2022 .

\section{Setting, sample size and population}

A purposive sample of 48 key informants will be recruited for Phase 1c (Q-methodology study) and Phase 2a (realist interviews and focus groups) (described below). Participants will comprise stroke survivors $(n=20)$, multidisciplinary community rehabilitation practitioners working with stroke survivors $(n=20)$ and team managers $(n=8)$.

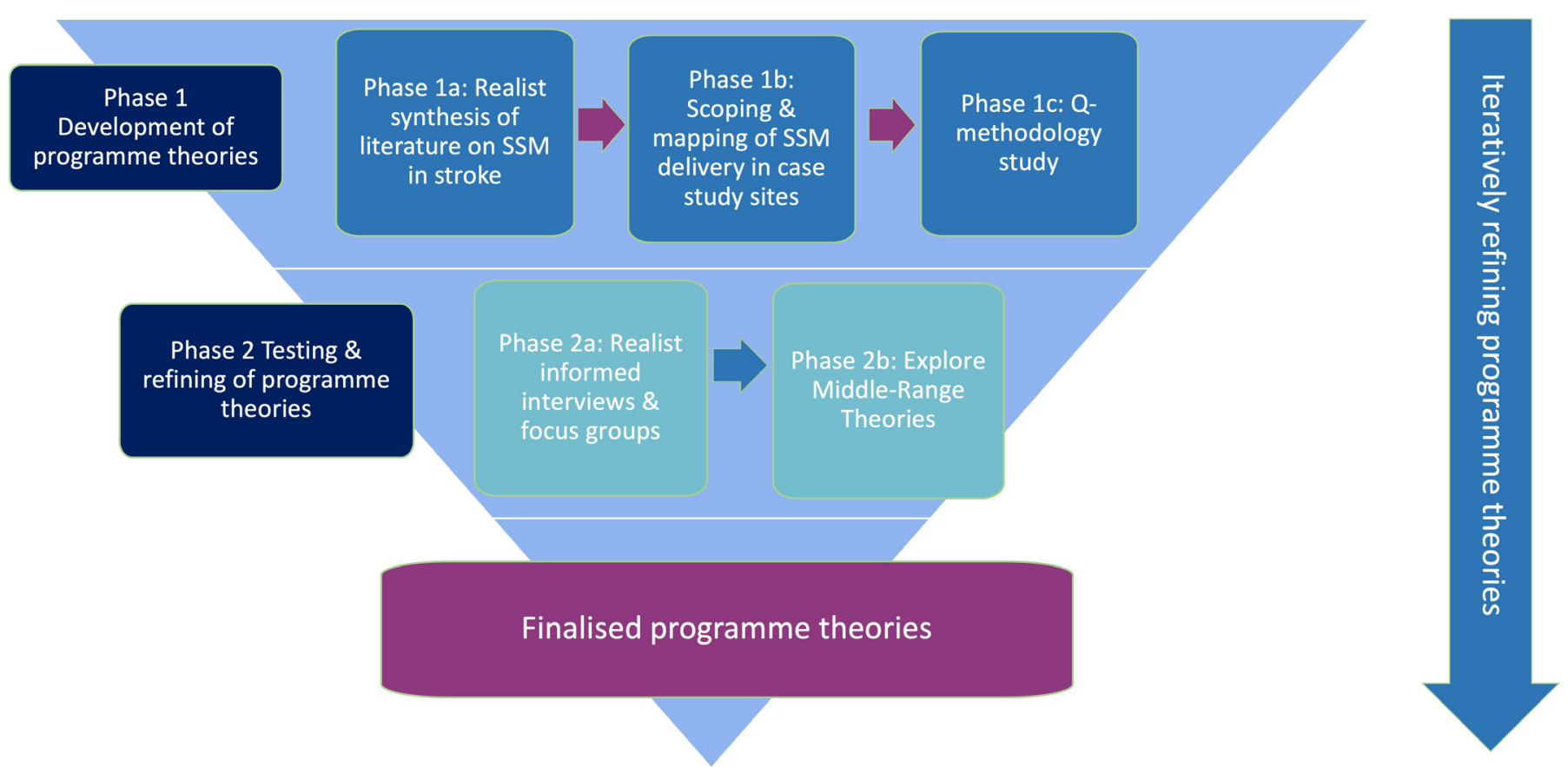

Figure 1 Outline of the stages of the study. SSM; Supported Self-Management. 
Table 2 Eligibility criteria

Stroke survivors

Men and women; aged $\geq 18$ years; with a diagnosis of stroke based on the WHO criteria; $\geq 6$ months poststroke; have been offered, received or engaged with SSM services (as self-reported by individual or by the local collaborator); are physically, cognitively and communicatively able to provide informed consent and participate; are sufficiently fluent in spoken and written English; residing in one of the case study sites and where the 'parent' clinical team feel that participation in the study is appropriate and safe.

\begin{tabular}{ll}
$\begin{array}{l}\text { Multidisciplinary } \\
\text { community }\end{array}$ & $\begin{array}{l}\text { Any multidisciplinary professional (eg, nurses, medics, physiotherapists, occupational therapists, } \\
\text { practitioners }\end{array}$ \\
$\begin{array}{ll}\text { psychologists, speech and language therapists, therapy assistants and social workers) who are identified } \\
\text { bSM services in one (or more) of the case study sites (including sign- posting to self-management } \\
\text { programmes or resources or see supported self-management as part of their role). }\end{array}$ \\
$\begin{array}{ll}\text { Managers/clinical } \\
\text { leads for stroke }\end{array}$ & $\begin{array}{l}\text { Any senior stakeholder (eg, clinical managers, clinical leads for stroke) who are identified by the local } \\
\text { collaborator/self-identify as having strategic responsibility for the organisation and delivery of community- } \\
\text { based SSM services in one (or more) of the case study sites. }\end{array}$ \\
\hline
\end{tabular}

SSM, Supported Self-Management.

Participants will be recruited across four case study sites in Scotland. Participants meeting the criteria outlined in table 2 will be recruited via a local collaborator (a stroke clinician or service/clinical network manager) based in each site. Local collaborators are independent from the project team (apart from in one case study site where the local collaborator is also a member of the project team). Four specific case study sites have been selected because these comprise different organisational structures and service delivery models for supported self-management in stroke; this includes stroke specialist, neurological and generic rehabilitation teams. Geographically, the four specific case study sites cover different areas of Scotland including remote and rural, and urban communities and caseloads with different socioeconomic characteristics. These case study sites will allow us to explore how contextual variation in geography, service structure and rehabilitation delivery models influence the mechanisms involved in the provision of community-based supported self-management for people who have had a stroke.

Realist and Q-methodology approaches rely on purposive sampling of participants who are likely to have insights and experiences relevant to developing the content and articulation of the programme theories. ${ }^{37-39}$ All of the interviewees in the current study will have experiences of receiving or delivering supported self-management, or its strategic oversight. Sample sizes are appropriate to the design of the study where in both realist and Q-methodology studies sample selection does not aim to achieve representativeness or saturation but aims to cover a range of views and contexts that might be relevant to the topic and the programme theories within the study. ${ }^{37-39}$ The sample sizes in the current study have been informed by the numbers of potentially eligible stroke survivors in each case study site, the number of clinicians and managers working in community teams in each site and the timelines for completion of the project.

The same interviewees will be invited to take part in both phases of the study. This design allows some flexibility, as Manzano $^{37}$ describes, in being able to revisit and explore initial ideas around the programme theories with interviewees as the research moves from the theory gleaning to theory testing stages. Although it is not expected, should there be a significant level of dropout between the phases of the project (between six to eight participants), ethical approval will be sought to recruit additional participants into the second phase of the study.

\section{Data collection and analysis}

The multiple phases and stages of the current study are summarised in table 3 .

Phase 1 (Phases 1a-1c): developing the initial programme theory Phase 1 aims to identify an initial set of programme theories, underpinned by $\mathrm{CMO}$ configurations to explain how, for whom and in what contexts community-based supported self-management might work. Phase 1 includes: a realist synthesis of primary and secondary research on supported self-management in stroke (Phase 1a); a scoping and mapping of how supported self-management is implemented across the case study sites involved in the study and the contexts in which this happens (Phase 1b); and a Q-methodology study to involve key stakeholders in the process of developing, refining and prioritising the initial programme theories (Phase 1c).

Further details on the realist synthesis approach taken in Phase $1 \mathrm{la}$ are described in PROSPERO. Briefly, however, in accordance with the RAMESES guidance ${ }^{40}$ Phase 1a begins with an initial set of informal ideas derived from previous research and discussions with key stakeholders about how supported self-management is intended to work in community rehabilitation settings. A preliminary search strategy (referred to as the 'parent search') will be designed to clarify key concepts and to help identify initial programme theories through the lens of potential contexts, mechanisms and outcomes of supported self-management interventions or approaches in stroke. Table 4 outlines the PICO criteria guiding the preliminary/parent search strategy. 
Table 3 Outline and description of the stages involved in the study

Phase Stage Methods

Phase 1a

Aim: To identify and describe initial programme theories and underpinning $\mathrm{CMOs}$
1. Initial ideas on 'what' supported self-management in stroke is and 'how' it is expected to work

2. Develop and run a preliminary 'parent' search of the literature on self-management +stroke

3. Describe specific context, mechanism and outcome configurations sitting underneath each programme theory

4. Initial appraisal (for relevance and rigour) and data extraction

5 Iterative and purposive searches, ongoing appraisal, journaling and data extraction in line with developing theories

Phase $1 b$

Aim: To describe the components, Scoping and mapping of the implementation of SSM in each structure, organisation and contexts case study site of delivery of supported selfmanagement approaches

\section{Phase 1c}

Aim: To validate, prioritise and

begin refining the initial theories and CMOs

\section{Phase 2a}

Aim: To test and consolidate the programme theories to produce robust, evidence-based explanatory framework

\section{Phase $2 \mathrm{~b}$}

Aim: To identify common patterns across the programme theories and provide an explanatory pathway of how SSM works

\section{Q-methodology study}

Seeking out and synthesis of middle range theories

\section{Methods}

Drawn from research team's previous research

Discussions with PPI group, stroke practitioners and researchers working in the field of stroke and selfmanagement research respectively Review of Blogs, Twitter, opinion pieces

Literature review of primary and secondary data on supported self-management in stroke table 4

Review of relevant papers that meet the inclusion criteria and appear to offer nuggets of information to help unpack the programme theories into CMOs

Journaling (writing a description of the paper, how it helps to inform or refine the programme theories/ CMOs, its overall quality in the sense of contributing to the developing programme theories) by way of appraisal of the relevance and rigour of the paper Extraction of nuggets of data and evidence relevant to developing programme theories, thematic analysis

Additional, purposive searches of specific bodies of literature that align directly with the programme theories to help develop these further (plus stage 4).

Bespoke proforma created to capture relevant information on structure, organisation and delivery of SSM in each site

\section{Q-sort statements}

Factor analysis

Qualitative follow-up interview comments

\section{Qualitative realist informed study Realist informed interviews}

Realist informed focus groups

Framework analysis

Middle range theory consolidation (apply MRTs based on relevance to explanatory power of programme theories)

Programme theory consolidation (confirm, refute or refine)

Additional literature search and synthesis (where relevant)

Narrative synthesis of evidence for which MRTs can be used to explain the programme theories.

CMO, context, mechanism and outcome; MRT, Mid-Range Theory; PPI, Patient and Public Involvement; SSM, Supported Self-Management.

Potentially useful peer-reviewed research papers will be identified and appraised for relevance and rigour through a process of 'journaling' using a bespoke appraisal form which aims to capture: (1) the characteristics of the documents/studies included, (2) the overall insights from the papers and (3) their quality (where appropriate). Papers will be categorised according to their relevance and appropriateness to the programme theories and nuggets of text from the papers, relevant to the underpinning CMOs, will be extracted. Where nuggets of text add new information or a new idea to the developing programme theories, these will be followed up with additional ad-hoc searches of more specific bodies of literature, as recommended. ${ }^{35} 41$ Although these steps in the synthesis appear linear, in practice, the iterative nature of programme theory development in realist studies means 
Table $4 \mathrm{PICO}(\mathrm{s})$ criteria guiding the preliminary search in Phase 1a

Participants/population Healthcare professionals caring for or involved in the provision of supported self-management approaches and interventions for, people (adults aged $>18$ years) with a diagnosis of stroke. Papers pertaining to people who do not have a diagnosis of stroke (eg, Transient Ischaemic Attack (TIA)), childhood stroke or which focus on informal caregivers only will be excluded.

Intervention(s)

Context

Outcome(s)

Types of study to be included

\section{Supported self-management refers to a collaborative approach which involves working in} partnership with stroke survivors and their families in an ongoing and sustained manner and includes interventions and approaches that help people to develop the skills, knowledge, confidence and resilience to manage the impact of their stroke. This includes aspects of medical, role and emotional management, behaviour change and the management of multiple morbidities. We are interested in papers that conceptualise supported self-management as an approach which embraces a paradigm shift in the way that practitioners, and stroke survivors and their families work together to jointly identify and address individuals' long-term needs, priorities and goals. We will exclude papers/ documents that do not align with this conceptualisation of supported self-management (ie, are about compliance with professionally driven treatment plans or supervision and instruction of rehabilitation exercises) or focus on general recovery, secondary prevention activities and rehabilitation in stroke (but with no reference to self-management approaches).

Papers identified in the systematic search will relate to the provision or experiences of supported selfmanagement in stroke in healthcare settings. Although community settings will be of specific interest, papers relating to other settings which can help to refine or refute the developing programme theories will be included.

The main outcome of this review is to identify programme theories that explain what works, for whom and under what circumstances and why in relation to the implementation of supported selfmanagement in stroke care.

In keeping with the RAMESES guidelines for realist reviews, ${ }^{47}$ all studies designs will be included. Documents such as editorials, opinion pieces, qualitative, quantitative and mixed-methods studies as well as systematic reviews may be included if they are relevant to the developing programme theories. Study protocols, conference abstracts and theses will be checked on Google Scholar for related full-text papers and where available, these will be retrieved and the original 'title' excluded. Where no alternative version exists, we will include the original 'title' if they provide evidence to support or refute the developing programme theories. that the steps overlap and are returned to as necessary in order to provide evidence to underpin the theories as they develop, until a degree of theoretical saturation in these is reached. ${ }^{41}$ It is expected that should anything 'new' be revealed during the testing of the programme theories in Phase 2a that further rounds of literature searching, appraisal and extraction will be undertaken in the synthesis and consolidation of these in Phase $2 b$ (figure 1).

Phase $1 \mathrm{~b}$ involves a scoping and mapping of how community-based supported self-management is currently organised and delivered across the case study sites in the study. A bespoke data collection proforma will be used by the researcher to gather data on: the stroke pathway from acute (hospital based care) to community; mechanisms of referral into community rehabilitation teams (eg, from stroke consultant, self-referral); number of referrals in total (and specifically for stroke if the team also treats other patients that is, is a generic team); the remit/model of community rehabilitation teams (stroke or generic); staffing (discipline, banding and static/rotational mix); how health and social care integration affects community team set up; frequency and type of multidisciplinary team meetings; if there is a cap on length of treatment or number of sessions provided to stroke survivors by members of the team; and formal supported self-management programmes in the area that staff are involved in or refer onto. The proforma has been created based on our existing knowledge of stroke care pathways and the provision of community rehabilitation services and will provide an understanding of the contexts, structure and organisation of supported self-management and its implementation in each community-based case study site.

Phase 1c comprises a Q-methodology study to involve key stakeholders in the process of developing and refining the initial programme theories (ahead of empirical testing in Phase 2). Q-methodology is a mixed-methods approach which allows us to identify and describe the shared viewpoints that exist on a topic, in this case supported self-management following stroke, revealing areas of consensus and disagreement. ${ }^{43}$ This is one of the first studies to use Q methodology to support a realist evaluation. Since the concept of supported self-management in stroke is still largely ill-defined, the Q-methodology study will specifically seek stakeholders' perceptions on the key strategies, mechanisms and outcomes of supported self-management in stroke to help prioritise the range of ideas underpinning the programme theories and to offer further insights to help with the articulation of wellformulated programme theories. 
Table 5 Stages of the Q-methodology study (Phase $2 \mathrm{a})^{38}$

\begin{tabular}{|c|c|}
\hline Stage & Summary of how this will be implemented in this study \\
\hline $\begin{array}{l}\text { 1. Selecting } \\
\text { the Q-set } \\
\text { (sample of card } \\
\text { statements) }\end{array}$ & $\begin{array}{l}\text { The statements used in a Q-study can be generated through a number of diverse sources. In this study, the } \\
\text { Q-set will be created from the emerging initial programme theories and potential contexts, mechanisms and } \\
\text { outcomes identified from the literature reviewed in Phase 1a. Q-sets can contain between } 30-80 \text { statements. } \\
\text { Examples of statements may include: Professionals decide what is best for the stroke survivor, flexibility and } \\
\text { freedom to decide what works best for me, understanding the person and their needs, being able to get } \\
\text { support from a wide range of local and community organisations. }\end{array}$ \\
\hline
\end{tabular}

2. Select the P- This stage involves the selection of participants who are considered to be 'information rich' (or key set (sample of informants) and likely to hold a range of viewpoints, and in this case, have insights and experiences relevant participants) to the developing programme theories. In this case, stroke survivors $(n=20)$, multidisciplinary communityrehabilitation practitioners $(n=20)$ and team managers $(n=8)$ will be recruited to participate in the $Q$ methodology study.

\begin{tabular}{|c|c|}
\hline $\begin{array}{l}\text { 3. The Q- } \\
\text { sorting process } \\
\text { (ranking of } \\
\text { the cards by } \\
\text { participants) } \\
\text { and brief } \\
\text { follow-up } \\
\text { interview }\end{array}$ & $\begin{array}{l}\text { In this stage, participants will be asked to rank the 'importance' of the statements according to a condition } \\
\text { of instruction, which in this case is in response to the question 'what is important to making supported self- } \\
\text { management work for you?' Participants will be asked to sort the cards into piles of agree, disagree and } \\
\text { neutral and then to rank them in order of importance on a bell-shaped Q-grid from most to least important. A } \\
\text { short follow-up interview is conducted with all participants to invite them to explain the position of their cards. } \\
\text { In this study, it is intended that these explanations will help to identify the importance of specific components } \\
\text { or structures involved in supporting self-management but also to provide insights into how contexts, } \\
\text { mechanisms and outcomes link together. }\end{array}$ \\
\hline $\begin{array}{l}\text { or } \\
\text { s and } \\
\text { etation }\end{array}$ & $\begin{array}{l}\text { Once the cards/statements have been sorted by participants, correlation and factor analysis is performed } \\
\text { using a statistical programme such as Ken-Q. The factor analysis identifies groups of participants who have } \\
\text { rank ordered the statements in a similar fashion and share similar perspectives. }{ }^{43} \text { A description of these } \\
\text { shared views are then presented for qualitative interpretation, in this case, shared views on what is important } \\
\text { for making supported self-management in stroke work and views on the most important and least important } \\
\text { statements in each 'factor'. This interpretation tells the story. }\end{array}$ \\
\hline
\end{tabular}

Table 5 outlines the four broad stages of a Q-methodology study $^{38}$ and how they apply in the current study. Briefly, ideas underpinning the initial programme theories generated in Phase 1 a will be used to inform a Q-set of statements. The statements will be printed onto cards and given to participants to rank from most to least important in relation to the question 'what is most important to make supported self-management work?'. The placing of the statements will be analysed through factor analysis using a programme called Ken-Q ${ }^{44}$ which helps to identify clusters of shared viewpoints across the participants. Participants will also be asked some follow-up interview questions to explain the reasons for why they positioned the cards. The resulting factors will be interpreted, drawing in the data from interview questions, and presented as narrative descriptions of the viewpoints. The framework of Q-methodology is a valuable approach for involving stakeholders in the theory development process since the combination of quantitative factor analysis and in-depth qualitative interpretation can foster deeper insight into the CMO configurations and overall programme theories. ${ }^{45}$

The final output of Phase 1 will be the articulation of a set of evidence-based initial programme theories (expressed as CMO configurations) to explain how supported self-management in community stroke rehabilitation might work, for whom and in which contexts.
Phase 2: testing, synthesis and consolidation of the programme theories to develop a robust explanatory model

Phase 2 aims to develop an explanatory model of how supported self-management works, for whom and in what contexts. Phase 2 involves: empirical testing and refinement of the programme theories (Phase 2a) and synthesis and consolidation of these through the lens of middle range theories (MRTs) to enhance the explanatory potential of the programme theories (Phase $2 \mathrm{~b}$ ).

In Phase 2a, individual interviews will be conducted with stroke survivors and team managers and focus groups will be undertaken with community rehabilitation team practitioners. Given COVID-19 restrictions at the time of the research, all data collection will be undertaken virtually by telephone and Microsoft Teams. In line with realistinformed interview principles, ${ }^{37}$ the interview guides will be specifically created to test the CMOs (from Phase 1) underpinning the programme theories with flexibility to allow participants to offer their own ideas. In a realist interview, the programme theories are shared with the interviewee for them to comment on these with a view to confirming or refuting their content, and essentially refining these. ${ }^{37}$ All interviews will be digitally recorded with consent and transcribed verbatim. Participants' consent will be reconfirmed prior to the start and at the end of the interviews and focus groups.

Data from Phase 2a will be analysed using Framework Analysis $^{46}$ where the programme theories and CMOs identified from Phase 1 will be used to develop the 
coding framework. Consistent with realist approaches, data analysis will be retroductive, ${ }^{47}$ applying both deductive and inductive logic to seek evidence to confirm and support existing programme theories and fresh information and ideas that help to construct new CMOs related to the overarching programme theories. Iterations to the programme theories and decision-making will be documented at each stage of the study enhancing the transparency of the approach and the robustness of the ensuing findings. NVivo V.12 will be used to help manage and synthesise the data from the interviews and focus groups and matrices charting the alignment of the qualitative evidence to the programme theories will be created to aid interpretation and refinement of the programme theories. Further rounds of literature searching, appraisal and extraction may also be undertaken at this point to provide evidence for any new ideas emerging from the qualitative data (figure 1).

The aim of Phase $2 b$ is to synthesise and consolidate the evidence underpinning the programme theories from across Phases 1 and 2 through the lens of relevant MRTs to develop a portable and robust explanatory model for how supported self-management works, for whom and in what contexts. The refined set of programme theories will help to explain how mechanisms and outcomes are linked to particular contexts and settings. However, situating these within relevant existing MRTs of behaviour or social change will help to elevate the granular, working programme theories to a more transferable understanding of how types of self-management interventions and approaches work in different types of circumstances. ${ }^{346}$ Appropriate MRTs will be identified through a purposive search for relevant, abstract theories drawing on (1) the work of scholars in the field of supported selfmanagement and behaviour change, (2) work undertaken in similar realist projects on self-management in other conditions and (3) the existing expertise of the project team in the areas of community and primary care for stroke survivors, supported self-management across LTCs and implementation science. Following previous guidance ${ }^{48}$ relevant MRTs will be selected according to the extent to which they offer guidance for explaining changes or behaviour at or between the micro, meso and macro levels, their potential fit with the aims of the current project, their potential for inspiring theory generation and their compatibility with realist principles.

The output of Phase 2 will be the articulation of a set of consolidated programme theories underpinned by a narrative account of the supporting evidence from across the two phases of the study and explored through the lens of appropriate MRTs, to explain how and why supported self-management in community stroke rehabilitation may work, for whom and in what contexts.

\section{Patient and public involvement}

Our approach to patient and public involvement (PPI) will be guided by the UK Standards for Public Involvement. ${ }^{49}$ A PPI group comprising four members who have previously had a stroke, was established at the grant writing phase. With regards to individual's preferences, the group members fulfil different roles, as outlined in Smits et $a \tilde{l}^{0}$ involvement matrix, including listener, co-thinker, advisor and decision-maker. The group have continued to provide guidance, expertise and support throughout the development of the research protocol and the initial stages of the research. The PPI group will continue to work with the research team throughout the project and provide help and guidance with dissemination and public engagement activities. PPI involvement throughout during the study will be reported in accordance with the Guidance for Reporting Involvement of Patients and the Public. ${ }^{51}$

\section{Advisory group}

An advisory group, comprising academics and clinicians working in the field of stroke, a stroke survivor and a representative from a national community and third sector organisation, was convened at the outset of the project to guide and support the research team, and monitor the progress of the study.

\section{ETHICS AND DISSEMINATION}

\section{Importance of the study}

The current study builds on and extends previous research in this area offering more depth, clarity and transferability of research findings on supported selfmanagement in stroke. Specifically, the current study aims to offer a nuanced understanding of how, for whom and why supported self-management in community stroke rehabilitation works. This is important for several reasons. First, an understanding of the enabling or disabling contexts and the mechanisms through which supported self-management approaches may work (or may not work) is useful for understanding transferability to other community settings. This will facilitate the development of context-specific recommendations to support, optimise, tailor and personalise the provision of supported self-management to the needs of the local context in which it is to be implemented, so that it meaningfully and effectively addresses the long-term priorities and outcomes of stroke survivors and their families in a sustainable way. Second, understanding the conditions and requirements for successful supported self-management helps to underpin the policy dialogue and design of evaluation strategies for supported selfmanagement so that this reflects a more authentic understanding of the diverse influences affecting the delivery and provision of supported self-management at a grassroots level. This understanding should contribute to robust, actionable and measurable policies and guidelines as a means to strengthen and support the delivery of high quality, person-centred, community-based supported selfmanagement. Finally, the evidence produced through this study will help to forge a shift in the evidence base on stroke self-management away from a focus on trials 
that simply address what works to broader approaches which identify how supported self-management works, informing the design, development and implementation of future community-based self-management approaches and interventions. From a realist perspective, the study will provide evidence on the value of Q-methodology as a novel approach for involving stakeholders in the development and prioritisation of programme theories.

\section{Ethics}

Ethical approval for the study was obtained from East of Scotland Research Ethics Committee (19/ES/0055) and Research \& Development approvals obtained from the participating NHS boards. The study commenced in March 2019 and was paused for 8 months from April 2020 to November 2020 because of the COVID-19 pandemic. Phase 1 has since been completed; data collection for Phase 2, all PPI and advisory group meetings and dissemination and impact activities will be conducted virtually for the remainder of the study.

\section{Dissemination and impact}

A final phase of the study will be to explore how best to articulate the project findings to different audiences and maximise the impact, influence and implementation of the findings within Scottish and UK stroke policy and community service provision. A potential limitation of the research is the Scottish context of the study, where healthcare is a devolved matter and is funded, organised and delivered differently from other parts of the UK. Scotland provides an exemplar for this research because the provision of supported self-management is mandated in stroke policy $^{52}$ and monitored through the Scottish Stroke Audit. ${ }^{27}$ However, understanding the transferability and implementation potential of the study findings across the UK will be important. Our project team members (MB, $\mathrm{KB}, \mathrm{TL}, \mathrm{MS}$ and RJF) hold strategic roles in the implementation of stroke policy and practice across the UK and are ideally placed to help facilitate dissemination, and to explore and maximise the impact of the findings within and beyond Scotland. Different dissemination and impact strategies will be required for this, and these will be refined as the project progresses.

Our broader dissemination strategy will involve online engagement events with stroke survivors and key stakeholders involved in the delivery of community-based stroke care across the UK, third sector support and stroke policy and commissioning. Regular project updates and summaries will be disseminated through our networks with academic, clinical and third sector partners, including The Stroke Association and the Health and Social Care Alliance's Self-Management Network and through our dedicated twitter page (@IMPETUS_stroke) which currently has over 339 followers. The findings will be disseminated more widely to clinical colleagues at practice-based events and professional journals, through professional forums such as UK Stroke Forum, Scottish Stroke Allied Health Professions Forum and the Health Services Research UK
Network. The findings will be disseminated to academic colleagues through presentations at national and international stroke-related conferences and publications in peer-reviewed journals. The findings will be disseminated to people affected by stroke through the UK Stroke Assembly and through the communication strategies of The Stroke Association and public engagement events. A plain English summary of the project and its findings will be sent to all study participants.

\section{Author affiliations}

${ }^{1}$ School of Medicine, Dentistry \& Nursing, University of Glasgow, Glasgow, UK ${ }^{2}$ School of Health and Life Sciences, Glasgow Caledonian University, Glasgow, UK ${ }^{3}$ Institute of Cardiovascular and Medical Sciences, University of Glasgow, Glasgow, UK

${ }^{4}$ Institute of Health and Wellbeing, University of Glasgow, Glasgow, UK

${ }^{5}$ Centre for Health and Social Care Research, St Georges University of London, London, UK

${ }^{6}$ Faculty of Medicine and Health Sciences, University of Nottingham, Nottingham, UK ${ }^{7}$ NHS Grampian, Woodend Hospital, Aberdeen, UK

${ }^{8}$ Scottish Government Stroke Improvement Programme, Edinburgh, UK

${ }^{9}$ Lanarkshire Acute Hospitals NHS Trust, Bothwell, UK

${ }^{10}$ NHS Lanarkshire, Coathill Hospital, Coatbridge, UK

${ }^{11} \mathrm{NHS}$ Lothian, Leith Community Treatment Centre, Leith, UK

Twitter Lisa Kidd @lisakidd22, Katie I Gallacher @GallacherKatie and Fiona Jones @JonesFio

Acknowledgements We wish to acknowledge and thank our patient and public involvement group members; Betty Gilmour, Colin Fraser, Pat Mooty and Audrey Taggart, and our advisory group members; Anne Armstrong, Professor Jo Booth, Professor Audrey Bowen, Phil Colis, Professor Helen Mason, Sara Redmond and Professor Frederike van Wijck (Chair) for their support and advice in shaping the study design and protocol, and for their continuing advice and guidance as the study progresses. We also wish to thank Dr Justin Jagosh from the Centre for Advancement in Realist Evaluation and Synthesis (CARES) for his methodological guidance and to Paul Cannon, College Librarian for Medical, Veterinary and Life Sciences, University of Glasgow, for his guidance in literature searching for Phase 1.

Contributors LK conceived the original study. HM, TQ, RJF, FJ, KIG, MB, KB, TL and MS contributed to the development of the research plans and original funding proposal. LK produced the first draft of this manuscript. LK, JDM, HM, TQ, RJF, FJ, $\mathrm{KIG}, \mathrm{MB}, \mathrm{KB}, \mathrm{TL}$ and $\mathrm{MS}$ contributed to reviewing and revising the manuscript. All authors read and approved the final version of the manuscript.

Funding This work was supported by The Stroke Association (Grant number SA PG 18\100067).

Competing interests None declared.

Patient and public involvement Patients and/or the public were involved in the design, or conduct, or reporting, or dissemination plans of this research. Refer to the Methods section for further details.

Patient consent for publication Not applicable.

Provenance and peer review Not commissioned; externally peer reviewed.

Open access This is an open access article distributed in accordance with the Creative Commons Attribution Non Commercial (CC BY-NC 4.0) license, which permits others to distribute, remix, adapt, build upon this work non-commercially, and license their derivative works on different terms, provided the original work is properly cited, appropriate credit is given, any changes made indicated, and the use is non-commercial. See: http://creativecommons.org/licenses/by-nc/4.0/.

ORCID iDs

Lisa Kidd http://orcid.org/0000-0003-2709-4011

Katie I Gallacher http://orcid.org/0000-0001-8620-8758

\section{REFERENCES}

1 The Stroke Association. State of the nation. London, UK, 2018. 
2 Gallacher KI, Batty GD, McLean G, et al. Stroke, multimorbidity and polypharmacy in a nationally representative sample of $1,424,378$ patients in Scotland: implications for treatment burden. BMC Med 2014;12:151.

3 Ayerbe L, Ayis S, Crichton S, et al. The long-term outcomes of depression up to 10 years after stroke; the South London stroke register. J Neurol Neurosurg Psych 2014;85:514-21.

4 World Health Organisation. Innovative care for chronic conditions: building blocks for action Geneva report. World Health Organisation, 2002.

5 Goodwin N, Curry N, Naylor C. Managing long-term conditions. London, UK: Kings Fund, 2010.

6 NHS England. The NHS long term plan department of health. London, UK, 2019.

7 NHS Scotland. Personalising realistic medicine Scottish government. Edinburgh, UK, 2019.

8 Rudd AG, Bowen A, Young G. National clinical guideline for stroke. London, UK: Royal College of Physicians, 2017.

9 Scottish Government. Scottish stroke improvement plan. Edinburgh, UK: Scottish Government, 2014.

10 Ellis J, Boger E, Latter S, et al. Conceptualisation of the 'good' self-manager: a qualitative investigation of stakeholder views on the self-management of long-term health conditions. Soc Sci Med 2017;176:25-33.

11 Parke HL, Epiphaniou E, Pearce G, et al. Self-management support interventions for stroke survivors: a systematic meta-review. PLoS One 2015;10:e0131448.

12 De longh A, Fagan P, Fenner J. A practical guide to self-management support London. The Health Foundation, 2015.

13 Jones F, Riazi A. Self-efficacy and self-management after stroke: a systematic review. Disabil Rehabil 2011:33:797-810.

14 Lennon S, McKenna S, Jones F. Self-management programmes for people post stroke: a systematic review. Clin Rehabil 2013;27:867-78.

15 Warner G, Packer T, Villeneuve M, et al. A systematic review of the effectiveness of stroke self-management programs for improving function and participation outcomes: self-management programs for stroke survivors. Disabil Rehabil 2015;37:2141-63.

16 Fryer CE, Luker JA, McDonnell MN. Self-management programmes for quality of life in people with stroke. Cochrane Library 2016;8:1-77.

17 Wray F, Clarke D, Forster A. Post-stroke self-management interventions: a systematic review of effectiveness and investigation of the inclusion of stroke survivors with aphasia. Disabil Rehabil 2018;40:1237-51.

18 Fu V, Weatherall M, McPherson K, et al. Taking charge after stroke: a randomized controlled trial of a person-centered, self-directed rehabilitation intervention. Int J Stroke 2020;15:954-64.

19 Boger EJ, Demain SH, Latter SM. Stroke self-management: a focus group study to identify the factors influencing self-management following stroke. Int J Nurs Stud 2015;52:175-87.

20 Kidd L, Lawrence M, Booth J, et al. Development and evaluation of a nurse-led, tailored stroke self-management intervention. BMC Health Serv Res 2015;15:359.

21 Satink T, Josephsson S, Zajec J, et al. Self-management develops through doing of everyday activities - a longitudinal qualitative study of stroke survivors during two years post-stroke. BMC Neurol 2016;16:221.

22 Clark E, Bennett K, Ward N, et al. One size does not fit all - Stroke survivor's views on group self-management interventions. Disabil Rehabil 2018;40:569-76.

23 Sadler E, Wolfe CDA, Jones F, et al. Exploring stroke survivors' and physiotherapists' views of self-management after stroke: a qualitative study in the UK. BMJ Open 2017;7:e011631.

24 McNaughton $\mathrm{H}$, Weatherall M, McPherson K, et al. The effect of the take charge intervention on mood, motivation, activation and risk factor management: analysis of secondary data from the taking charge after stroke (TaCAS) trial. Clin Rehabil 2021;35:269215521993648

25 Jones F, McKevitt C, Riazi A, et al. How is rehabilitation with and without an integrated self-management approach perceived by UK community-dwelling stroke survivors? A qualitative process evaluation to explore implementation and contextual variations. BMJ Open 2017;7:e014109.

26 Kidd L, Booth J, Lawrence M, et al. Implementing supported selfmanagement in community-based stroke care: a secondary analysis of nurses' perspectives. J Clin Med 2020;9:985.
27 NHS National Services Scotland. Scottish stroke improvement programme report. Edinburgh: ISD Scotland, 2016.

28 Gallacher K, Morrison D, Jani B, et al. Uncovering treatment burden as a key concept for stroke care: a systematic review of qualitative research. PLoS Med 2013;10:e1001473.

29 Gallacher KI, May CR, Langhorne P, et al. A conceptual model of treatment burden and patient capacity in stroke. BMC Fam Pract 2018;19.

30 Kulnik ST, Pöstges H, Brimicombe L, et al. Implementing an interprofessional model of self-management support across a community workforce: a mixed-methods evaluation study. $J$ Interprof Care 2017;31:75-84.

31 Pawson R, Tilley N. Realistic evaluation. London; UK: Sage Ltd, 1997.

32 Wong G, Westhorp G, Manzano A, et al. RAMESES II reporting standards for realist evaluations. BMC Med 2016;14:96.

33 Westhorp G. Realist impact evaluation: an introduction. London, UK: Overseas Development Institute, 2014.

34 Westhorp G. Chapter 3: Understanding mechanisms in realist evaluation and research. In: Emmel N, Greenhalgh J, Manzano A, et al, eds. Doing realist research. London, UK: Sage Ltd, 2018: 41-57.

35 Greenhalgh J, Pawson R, Wright J, et al. Functionality and feedback: a protocol for a realist synthesis of the collation, interpretation and utilisation of PROMs data to improve patient care. BMJ Open 2014;4:e005601.

36 Punton M, Vogel I, Leavy J. Reality bites: making realist evaluation useful in the real world. In: Centre for development impact practice paper. , 2020: 22, 1-13.

37 Manzano A. The craft of interviewing in realist evaluation. Evaluation 2016;22:342-60.

38 Baker R, Thompson C, Mannion R. Q methodology in health economics. J Health Serv Res Policy 2006;11:38-45.

39 Emmel N. Sampling and choosing cases in qualitative research: a realist approach. London, UK: Sage, 2013.

40 RAMESES II Project. Developing realist programme theories [online], 2017. Available: http://www.ramesesproject.org/media/RAMESES II_Developing_realist_programme_theories.pdf [Accessed $21 \mathrm{Mar}$ 2020]

41 Wong G, Greenhalgh T, Westhorp G. RAMESES publication standards: realist syntheses. BMC Medicine 2013;11.

42 Blane DN, Macdonald S, Morrison D, et al. Interventions targeted at primary care practitioners to improve the identification and referral of patients with co-morbid obesity: a realist review protocol. Syst Rev 2015;4:61.

43 Watts S, Stenner P. Doing Q ethodology: theory, method and interpretation. Qual Res Psychol 2005;2:67-91.

44 Banasick S. Ken-Q Analysis (Version 1.0.6) [Software], 2019. Available: https://shawnbanasick.github.io/ken-q-analysis-beta/ index.html\#section1 [Accessed Jun 2021].

45 Harris K, Henderson S, Wink B. Mobilising Q methodology within a realist evaluation: lessons from an empirical study. Evaluation 2019;25:430-48.

46 Richie J, Spencer L. Qualitative data analysis for applied policy research. In: Bryman B, Burgess RG, eds. Analysing qualitative data. London, UK: Routledge, 2002: 173-94.

47 RAMESES II Project. Retroduction in realist evaluation [online], 2017. Available: http://www.ramesesproject.org/media/RAMESES_II_ Retroduction.pdf[Accessed 21 Mar 2021].

48 Shearn K, Allmark P, Piercy H, et al. Building realist program theory for large complex and messy interventions. Int J Qual Methods 2017:16:160940691774179.

49 National Institute for Health Research/UK Public Involvement Standards Development Partnership Group. UK standards for public involvement: better public involvement for better health and social care. Southampton: NIHR, 2019.

50 Smits D-W, van Meeteren K, Klem M, et al. Designing a tool to support patient and public involvement in research projects: the involvement matrix. Res Involv Engagem 2020;6:30.

51 Staniszewska S, Brett J, Simera I, et al. GRIPP2 reporting checklists: tools to improve reporting of patient and public involvement in research. BMJ 2017;358:j3453.

52 Scottish Government. Stroke improvement plan. Edinburgh: Scottish Government, 2014. https://www.gov.scot/publications/strokeimprovement-plan/ 